\section{Are patients with rheumatoid arthritis prone to accelerated atherosclerosis?}

Whether preclinical or early signs of atherosclerosis are more prevalent in patients with rheumatoid arthritis (RA) in unclear. In a cross-sectional study, Roman and colleagues at the Hospital for Special Surgery, NY, sought to establish the prevalence of atherosclerosis in 98 consecutive outpatients with RA and 98 controls matched for age, sex and ethnicity.

Carotid ultrasonography was carried out in all patients, as were inflammatory marker assays and evaluation of cardiovascular risk factors such as hypertension, smoking and low serum levels of HDL cholesterol.

Compared with the controls, the prevalence of carotid atherosclerosis was three times higher in patients with RA, despite these patients having more-favorable risk factor profiles. Age, hypertension and therapy with tumor necrosis factor- $\alpha$ inhibitors were the only risk factors that correlated significantly with an increased likelihood of atherosclerotic plaque.

Limitations of the study included its cross-sectional design, use of a single set of inflammatory marker measurements and incomplete data on lifetime doses of medications. As chronic inflammation is likely to have a role in the early onset of atherosclerosis in patients with RA, the authors emphasize the need for prospective management of cardiac risk factors in addition to aggressive RA therapy.

\section{Claire Braybrook}

Original article Roman MJ et al. (2006) Preclinical carotid atherosclerosis in patients with rheumatoid arthritis. Ann Intern Med 144: 249-256

\section{Denosumab improves low BMD in postmenopausal women}

Denosumab is a fully human monoclonal antibody that targets RANKL (the receptor activator of nuclear factor $\mathrm{kB}$ ligand) - a protein that acts as the primary mediator of osteoclast differentiation, activity, and lifespan. In a phase II, randomized, placebo-controlled study, McClung et al. evaluated the efficacy of denosumab in postmenopausal women younger than 80 years who had a low BMD.

In total, 369 women from 29 study centers in the US completed this 12-month study. Participants were randomly assigned to receive subcutaneous denosumab, either every 3 months $(6,14$, or $30 \mathrm{mg})$ or every 6 months $(4,60,100$, or $210 \mathrm{mg}$ ), open-label $70 \mathrm{mg}$ alendronate per week, or placebo. At 12 months, women who received denosumab had a mean increase in BMD of $3.0-6.7 \%$ at the lumbar spine, compared with a $4.6 \%$ increase and a $0.8 \%$ decrease in women who received alendronate and placebo, respectively. Women who received denosumab also had a greater increase in total hip BMD and BMD in the distal third of the radius compared with the alendronate and placebo groups. Levels of serum C-TELOPEPTIDE decreased as early as 3 days in women who received denosumab. A dose of $30 \mathrm{mg}$ denosumab every 3 months, or $60 \mathrm{mg}$ every 6 months seemed to be the most effective regimens. Adverse events were not significantly different among the three treatment arms.

The authors conclude that denosumab administered subcutaneously to postmenopausal women causes a decrease in bone turnover and an increase in BMD. Denosumab might, therefore, be an effective treatment for osteoporosis.

Marie Lofthouse

Original article McClung MR et al. (2006) Denosumab in postmenopausal women with low bone mineral density. N Engl J Med 354: 821-831
GLOSSARY

C-TELOPEPTIDE

Type I collagen breakdown product that is used as a marker of bone resorption 